\title{
Dexmedetomidine enhances tolerance to bupivacaine cardiotoxicity in the isolated rat hearts: alpha 2 adrenoceptors were not involved
}

Fangfang $\mathrm{Xia}^{\dagger} \mathbb{D}$, Zhousheng Jin ${ }^{\dagger}$, Tingting Lin, Xixi Cai, Linmin Pan, Shi Wang, Yaoyao Cai and Hongfei Chen ${ }^{*}$ (D)

\begin{abstract}
Background: Dexmedetomidine was proved to mitigate bupivacaine-induced cardiotoxicity but mechanism of this ability is still unclear. This study was designed to investigate the direct effects of dexmedetomidine on cardiotoxicity induced by bupivacaine on Langendorff rat heart preparation and the role of alpha 2 adrenoceptors in this process was explored.

Methods: Hearts of rat were isolated, mounted on a Langendorff system. Five experimental groups were assessed after 10 min Krebs-Henseleit buffer (KHB) infusions as follow: (1) Group Con, only KHB was perfused; (2) Group Dex, KHB was perfused for 5 min, then dexmedetomidine $(10 \mathrm{nmol} / \mathrm{L}$ ) was added; (3) Group Bupi, KHB was perfused for $25 \mathrm{~min}$, then bupivacaine $(50 \mu \mathrm{mol} / \mathrm{L})$ was added; (4) Group Bupi + Dex, KHB was perfused for $5 \mathrm{~min}$, then the dexmedetomidine $(10 \mathrm{nmol} / \mathrm{L})$ was added for $20 \mathrm{~min}$, at last a mixture of $\mathrm{KHB}+$ dexmedetomidine + bupivacaine were perfused; (5) Group Bupi + Dex + Yoh, a combination of KHB + yohimbine (alpha 2 adrenoceptor antagonists, $1 \mu \mathrm{mol} / \mathrm{L})$ was perfusion for $5 \mathrm{~min}$, then dexmedetomidine $(10 \mathrm{nmol} / \mathrm{L})$ was added for $20 \mathrm{~min}$, at last a mixture of $\mathrm{KHB}+$ yohimbine + dexmedetomidine + bupivacaine was perfused. The experimental perfusion was maintained for 35 min in group Con and group Dex, and the experimental perfusion was sustained until asystole in the other three groups.

Results: Compared with group Bupi, dexmedetomidine significantly increased the time to first arrhythmia $(P<0.001)$ and time to asystole $(P<0.001)$ in group Bupi + Dex. In addition, dexmedetomidine also significantly increased the time to 25,50 and $75 \%$ reductions in heart rate $(P<0.001)$ and the time to 25,50 and $75 \%$ reductions in rate-pressure product $(P<0.001)$ in group Bupi + Dex. Dexmedetomidine increased the cardiac tissue bupivacaine content when asystole (Bupi + Dex vs. Bupi, $58.5 \pm 6.3$ vs. $46.8 \pm 5.6 \mathrm{nmol} / \mathrm{g}, P=0.003$ ). The benefit of dexmedetomidine on bupivacaine-induced cardiotoxicity were not eliminated by yohimbine.

Conclusions: Dexmedetomidine could delay the occurrence of bupivacaine-induced arrhythmia and asystole in the isolated rat hearts, but the alpha 2 adrenoceptors were not involved in this process.
\end{abstract}

Keywords: Dexmedetomidine, Bupivacaine, Cardiotoxicity, Alpha 2 adrenoceptors, Isolated heart

\footnotetext{
*Correspondence: chhfei@126.com

${ }^{\dagger}$ Fangfang Xia and Zhousheng Jin contributed equally to this work, and

Zhousheng Jin should be considered co-first authors.

Department of Anesthesiology, The First Affiliated Hospital of Wenzhou

Medical University, Shangcai village, Nanbaixiang town, Ouhai District,

Wenzhou City 325000, Zhejiang Province, China
}

(c) The Author(s). 2019 Open Access This article is distributed under the terms of the Creative Commons Attribution 4.0 International License (http://creativecommons.org/licenses/by/4.0/), which permits unrestricted use, distribution, and reproduction in any medium, provided you give appropriate credit to the original author(s) and the source, provide a link to the Creative Commons license, and indicate if changes were made. The Creative Commons Public Domain Dedication waiver (http://creativecommons.org/publicdomain/zero/1.0/) applies to the data made available in this article, unless otherwise stated. 


\section{Background}

Bupivacaine is a long-acting, lipophilic, amide class local anesthetic commonly used to provide local anesthesia during surgical procedures in clinical practice because of its high analgesic potency and long-lasting effects. Unfortunately, bupivacaine exhibits a strong tendency to cardiotoxicity when unintentional intravenous injection or overdose [1]. Cardiotoxicity induced by bupivacaine, which can lead to arrhythmias, poor myocardial contractility and even cardiac arrest, is difficult to reverse [2].

Dexmedetomidine, a highly selective $\alpha-2$ adrenoceptor agonist with a broad range of pharmacological properties, which is widely used for its sedative effects clinically $[3,4]$. Accumulating evidence suggests that dexmedetomidine could be used as a local anesthetics adjuvant to enhance the analgesic effect [5-7], and also provide sedation [5]. Hanci et al. [8] reported dexmedetomidine pretreatment could strengthen tolerance to bupivacaineinduced cardiotoxicity in rats. It demonstrated dexmedetomidine $(10 \mu \mathrm{g} / \mathrm{kg})$ pretreatment increased the time to decreases in heart rate, reductions in mean arterial pressure, first arrhythmia and asystole. Hanci suspected that dexmedetomidine's ability to mitigate bupivacaineinduced cardiotoxicity may be related with its sympatholytic properties [9] and anti arrhythmogenic [10]. However, the action sites of dexmedetomidine mitigate local anesthetics-induced cardiotoxicity has not yet been delineated.

Our primary hypothesis was that dexmedetomidine can mitigate cardiotoxicity of bupivacaine by acting directly on the isolated heart. In this study, we established bupivacaine cardiotoxicity model in the isolated rat hearts and investigated the direct effects of dexmedetomidine on cardiotoxicity induced by bupivacaine. The main indicators in our experiment are the time to the first arrhythmia $\left(\mathrm{T}_{\text {arrhythmia }}\right)$ and the time to asystole $\left(\mathrm{T}_{\text {asystole }}\right)$, secondary indicators include the cardiac tissue bupivacaine content when asystole.

\section{Methods}

\section{Animals}

All animal protocols were approved by the Wenzhou Medical University Animal Care and Use Committee (wydw 2015-0121, Zhejiang, China). Adult male Sprague-Dawley rats (SYXK 2015-0150), weighing between 320 and $380 \mathrm{~g}$ were provided by the Animal Center of Wenzhou Medical University. The care and handling of animals were in accordance with National Institutes of Health guidelines. All animal's body were incinerated after the study by the Animal Center of Wenzhou Medical University. A completed ARRIVE (Animal Research: Reporting of In Vivo Experiments) guidelines checklist is included in Checklist.

\section{Drugs}

Bupivacaine hydrochloride (0.5\%, Sigma-Aldrich Co., St. Louis, MO, P code: 101524503 B5274-5G), Dexmedetomidine (Sigma-Aldrich Co., St. Louis, MO, P code: 12815 SML0956-10MG) and Yohimbine (Sigma-Aldrich Co., St. Louis, MO, P code: 101509939 Y3125-1G) were used.

\section{Preparation of isolated hearts}

The isolated perfused, nonrecirculating Langendorff rat heart preparation was used in our study, as described previously [11]. Rats were anesthetized by the intraperitoneal injection of $80 \mathrm{mg} / \mathrm{kg}$ ketamine hydrochloride and $12 \mathrm{mg} / \mathrm{kg}$ xylazine, then $1000 \mathrm{U} / \mathrm{kg}$ heparin to prevent the formation of intracoronary microthrombi. The rats were euthanized by cervical dislocation, then the hearts were rapidly excised and perfused via the coronary arteries by tying the aorta onto a cannula (ML870B2, AD Instruments, Australia). The constant perfusion pressure was $80 \mathrm{mmHg}$, and a modified Krebs-Henseleit buffer (KHB) was used and described as follows: $\mathrm{NaCl} 118$ $\mathrm{mmol} / \mathrm{L}, \mathrm{KCl} 4.7 \mathrm{mmol} / \mathrm{L}, \mathrm{MgSO}_{4} 1.2 \mathrm{mmol} / \mathrm{L}, \mathrm{KH}_{2} \mathrm{PO}_{4}$ $1.2 \mathrm{mmol} / \mathrm{L}, \mathrm{NaHCO}_{3} 25.0 \mathrm{mmol} / \mathrm{L}, \mathrm{CaCl}_{2} 2.5 \mathrm{mmol} / \mathrm{L}$, glucose $10 \mathrm{mmol} / \mathrm{L}$. The solution was continuously bubbled with $95 \% \mathrm{O}_{2}$ and $5 \% \mathrm{CO}_{2}$, and $\mathrm{pH}$ was maintained at $7.40 \pm 0.05$. The left ventricular pressure was continuously monitored by a latex balloon placed in the left ventricle. Saline was intermittently injected into the balloon to maintain the left ventricular end-diastolic pressure at 4-10 $\mathrm{mmHg}$. Electrocaridiograph (ECG) electrodes were consistently placed in a "leadII" position. All data were collected using a PowerLab biological signal processing and analysis system (ML870, Australia Ad Instruments) and the Chart 5.5 .6 biological signal recording software. The experimental protocol was started after the $10 \mathrm{~min}$ of $\mathrm{KHB}$ as the steady-state baseline conditions.

\section{Experimental protocol}

Forty isolated rat hearts were mounted on the Langendorff system and then randomly assigned to 5 groups (Fig. 1): Group Con, Group Dex, Group Bupi, Group Bupi + Dex and Group Bupi + Dex + Yoh $(n=8)$. The KHB was continuously perfused into the hearts in all groups until the end. Experimental perfusion was started according to the assigned group after steady state was reached. In group Con, only $\mathrm{KHB}$ was perfused; In group Dex, dexmedetomidine and $\mathrm{KHB}$ were perfused; In group Bupi, KHB was perfused $25 \mathrm{~min}$ and then $50 \mu \mathrm{mol} / \mathrm{L}$ bupivacaine was added, whereas in group Bupi + Dex, $10 \mathrm{nmol} / \mathrm{L}$ dexmedetomidine was added after $5 \mathrm{~min}$ of $\mathrm{KHB}$ perfusion, and $50 \mu \mathrm{mol} / \mathrm{L}$ bupivacaine was added 20 min later. Besides, in group Bupi + Dex + Yoh, $1 \mu \mathrm{mol} / \mathrm{L}$ yohimbine was added immediately after the steady state, then $10 \mathrm{nmol} / \mathrm{L}$ dexmedetomidine 


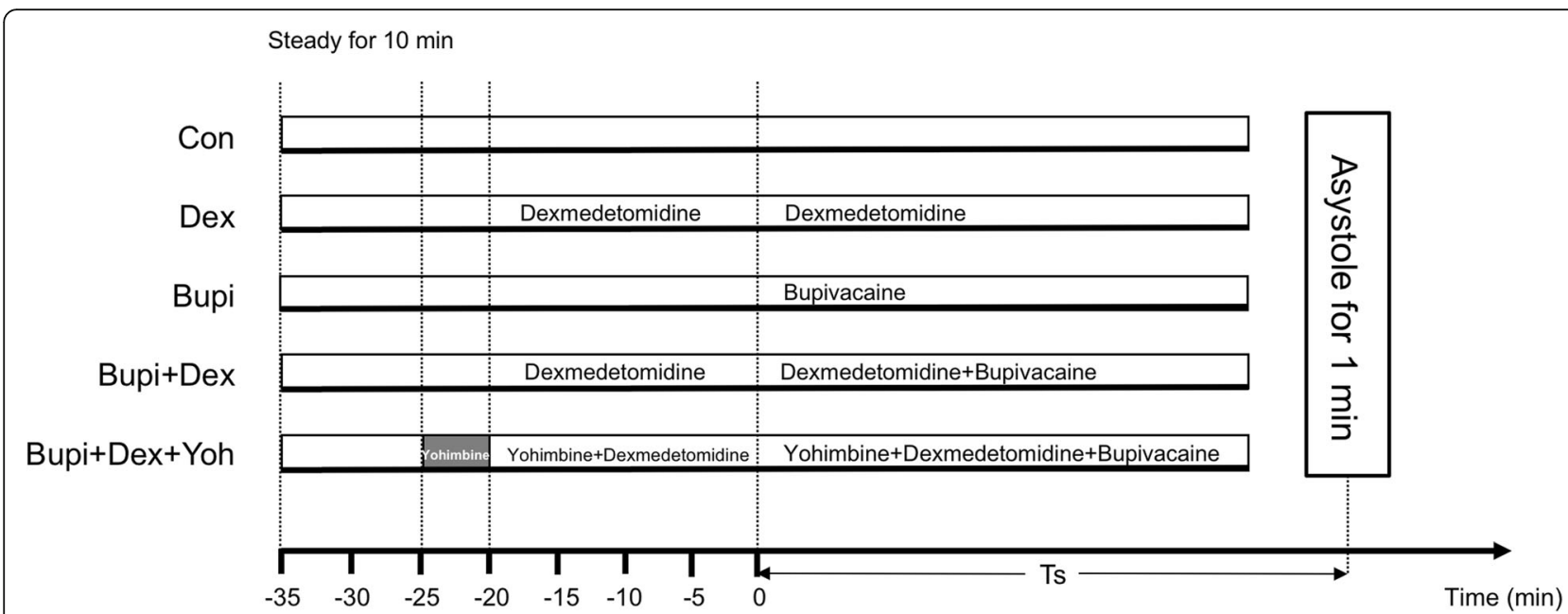

Fig. 1 Experimental Protocol ( $n=8$ in each group). Con, control; Bupi, bupivacaine; Dex, dexmedetomidine; Yoh, yohimbine. Without the infusion of bupivacaine, there was no arrhythmia or asystole occurred in group Con and group Dex. As a result, there was no values for $T_{\text {arrhythmia }} T_{\text {asystole, }}$ the time of reductions in HR and RPP in this table

was added $5 \mathrm{~min}$ later, and $50 \mu \mathrm{mol} / \mathrm{L}$ bupivacaine was added after 20 min later. In all groups but the group Con and group Dex, the experimental perfusion was sustained until asystole, and group Con and group Dex were perfused for $35 \mathrm{~min}$. The recorded data were evaluated and the following times were documented for each heart in all groups: the time of the first premature ventricular contraction accompanied by abnormal systole on the pressure trace after the bupivacaine perfusion ( $\mathrm{T}_{\text {arrhythmia }}$, Time to the first arrhythmia); the time from the initiation of $50 \mu \mathrm{mol} / \mathrm{L}$ bupivacaine infusion to asystole ( $>1 \mathrm{~min})\left(\mathrm{T}_{\text {asystole }}\right.$, Time to asystole); time to 25,50 and $75 \%$ reductions in heart rate (HR) and rate-pressure product $(\mathrm{RPP}=\mathrm{HR} \times($ systolic pressure diastolic pressure)) relative to baseline. Baseline values of hemodynamic were obtained after the $10 \mathrm{~min}$ with only KHB perfusion.

\section{Cardiac tissue bupivacaine content [12]}

After asystole, heart apex was cut immediately and stored in liquid nitrogen. Frozen hearts were grinded in a precooling glass tissue grinder with ice and homogenized with $0.4 \mathrm{~mol} / \mathrm{L}$ perchloric acid by $10 \mathrm{~mL} / \mathrm{g}$. Precipitated proteins were separated by centrifugation at $4000 \mathrm{~g}$ for $15 \mathrm{~min}$, and the supernatant was neutralized with 2 $\mathrm{mol} / \mathrm{L} \mathrm{KOH}$ to adjust $\mathrm{pH}$ at $6.0-7.0$ by $\mathrm{pH}$ meter. Samples were then centrifuged at $4000 \mathrm{~g}$ for another $15 \mathrm{~min}$ and the supernatant was filtered through a $0.22 \mu \mathrm{m}$ filter to determine the sample. The above operation was completed at $4{ }^{\circ} \mathrm{C}$. The concentration of bupivacaine in cardiac tissue was determined by HPLC-MS/MS (High performance liquid chromatography-mass spectrometry/ mass spectrometry).

\section{Statistical analysis}

The power analysis was based on our preliminary study which is mentioned above using the Power Sample Size (PASS 11.0) software program. We compared $T_{\text {arrhythmia }}$ among the various treatment groups. In our preliminary study, we totally used 9 rats: 3 rats in each of 3 groups. The means \pm standard deviation(SD) of $\mathrm{T}_{\text {arrhythmia }}$ in Bupi, Bupi + Dex and Bupi + Dex + Yoh groups were $161.0 \pm 4.9,283.7 \pm 15.2$, and $283.3 \pm 5.9$, respectively. Using a two-tailed type one error at $5 \%$ and type two error of $10 \%(\alpha=0.05, \beta=$ $0.1)$, the sample size of 6 per group was obtained. Balancing this fact with the desire to reduce the use of animals, we enrolled 8 rats per group for the study. At this point in the development of an assay sample sizing should only be viewed as a guide because the estimates of both the effect size and associated variability are approximate and come with much uncertainty attached to them.

SPSS (version 19.0, Chicago, IL) was used to carry out the computations. The measurement data were tested for normality using the Shapiro-Wilk test, normally distributed data were presented as the means $\pm \mathrm{SD}$. Oneway ANOVA was used to evaluate differences among groups, and the Tukey test was used as a post hoc test when significance was achieved. Statistical significance was considered as $P<0.05$.

\section{Results}

There were no differences in baseline weight (351.1 \pm $15.3, \quad 342.9 \pm 17.5, \quad 353.5 \pm 19.2, \quad 352.3 \pm 21.2, \quad 349.1 \pm$ $22.3 \mathrm{~g}$ for groups Con, Dex, Bupi, Bupi + Dex and Bup + Dex + Yoh, respectively), baseline HR (300.8 \pm 13.6, $297.6 \pm 19.2, \quad 296.3 \pm 14.0, \quad 298.0 \pm 21.3, \quad 299.4 \pm$ 
19.8 beats/min, respectively), baseline RPP $(36,788.8 \pm$ $2431.2,36,810.8 \pm 2731.6,36,096.1 \pm 1863.2,36,452.4 \pm$ $2670.2, \quad 37,456.3 \pm 3125.5 \mathrm{mmHg} \cdot$ beats/min, respectively) among the 5 groups in the $\operatorname{study}(P>0.05)$. No obvious adverse reactions occurred throughout the experiment.

\section{$T_{\text {arrhythmia }}$ and $T_{\text {asystole }}$}

Table 1 showed that the $\mathrm{T}_{\text {arrhythmia }}$ in the Bupi + Dex and Bupi + Dex + Yoh groups were comparable $(P=0.998)$, but both were significantly longer than the Bupi group $(P<0.001, P<0.001$, respectively). Similarly, there was no difference in the $\mathrm{T}_{\text {asystole }}$ between the Bupi + Dex and Bupi + Dex + Yoh groups $(P=0.990)$, but both $\mathrm{T}_{\text {asystole }}$ were significantly longer than that in the Bupi group $(P<0.001, P<0.001$, respectively).

\section{Cardiac function variables---- HR, RPP}

The HRs and RPPs in group Con showed no difference with the baseline throughout the experiment $(P>0.05)$. After infusion of dexmedetomidine, the HRs and RPPs also showed no statistical differences between group Dex and group Con $(P>0.05)$. (Figs. 2 and 3$)$.

The time to 25,50 and $75 \%$ reductions in HR and the time to 25,50 and $75 \%$ reductions in RPP were significantly increased in group Bupi + Dex than those in group Bupi $(P<0.001$ for all comparisons, Table 1$)$. The time to 25,50 and $75 \%$ reductions in $\mathrm{HR}$ and the time to 25, 50 and $75 \%$ reductions in RPP in group Bupi + Dex + Yoh were significantly increased than those in group Bupi $(P<0.001$ for all comparisons, Table 1), but were no statistical difference with group Bupi $+\operatorname{Dex}(P>0.05$ for all comparisons, Table 1). HR was significantly lower in group Bupi than in the Bupi + Dex and Bupi + Dex + Yoh groups for all measurements from 2 to $6 \mathrm{~min}$ after the bupivacaine infusion $(P<0.05)$. RPP was significantly lower in group Bupi than that in the Bupi + Dex and Bupi+ Dex + Yoh groups for all measurements from 1 to $6 \mathrm{~min}$ after the bupivacaine infusion $(P<0.05)$. HRs and RPPs values showed no differences between the groups Bupi + Dex and Bupi + Dex + Yoh $(P>0.05)$.

Table 1 Times to defined end points in the HR, RPP and ECG after bupivacaine perfusion

\begin{tabular}{|c|c|c|c|c|c|c|c|}
\hline Times to event (seconds) & Con & Dex & Bupi & Bupi + Dex & Bupi + Dex + Yoh & & $p$ \\
\hline \multirow[t]{3}{*}{$T_{\text {arrhythmia }}$} & - & - & $160.9 \pm 23.5$ & $277.8 \pm 27.1$ & $278.1 \pm 31.2$ & Con vs. Bupi+Dex & $<0.001$ \\
\hline & & & & & & Con vs. Bupi+Dex Yoh+ & $<0.001$ \\
\hline & & & & & & Dex vs. Yoh + Dex & 0.100 \\
\hline \multirow[t]{3}{*}{$\mathrm{T}_{\text {asystole }}$} & - & - & $360.3 \pm 11.5$ & $546.1 \pm 23.8$ & $543.16 \pm 37.2$ & Con vs. Dex & $<0.001$ \\
\hline & & & & & & Con vs. Yoh + Dex & $<0.001$ \\
\hline & & & & & & Dex vs. Yoh + Dex & 0.099 \\
\hline \multirow[t]{3}{*}{$\mathrm{HR}, 25 \%$ reduction } & - & - & $131.8 \pm 14.0$ & $179.8 \pm 24.8$ & $180.3 \pm 14.8$ & Con vs. Dex & $<0.001$ \\
\hline & & & & & & Con vs. Yoh + Dex & $<0.001$ \\
\hline & & & & & & Dex vs. Yoh + Dex & 0.096 \\
\hline \multirow[t]{3}{*}{ HR, 50\% reduction } & - & - & $257.1 \pm 26.6$ & $326.5 \pm 23.7$ & $330.6 \pm 28.5$ & Con vs. Dex & $<0.001$ \\
\hline & & & & & & Con vs. Yoh + Dex & $<0.001$ \\
\hline & & & & & & Dex vs. Yoh + Dex & 0.097 \\
\hline \multirow[t]{3}{*}{$H R$, $75 \%$ reduction } & - & - & $303.1 \pm 20.6$ & $418.1 \pm 57.0$ & $424.3 \pm 27.2$ & Con vs. Dex & $<0.001$ \\
\hline & & & & & & Con vs. Yoh + Dex & $<0.001$ \\
\hline & & & & & & Dex vs. Yoh + Dex & 0.080 \\
\hline \multirow[t]{3}{*}{ RPP, $25 \%$ reduction } & - & - & $121.9 \pm 15.8$ & $165.5 \pm 14.3$ & $160.6 \pm 13.4$ & Con vs. Dex & $<0.001$ \\
\hline & & & & & & Con vs. Yoh + Dex & $<0.001$ \\
\hline & & & & & & Dex vs. Yoh + Dex & 0.099 \\
\hline \multirow[t]{3}{*}{ RPP, $50 \%$ reduction } & - & - & $223.6 \pm 25.5$ & $316.4 \pm 23.4$ & $321.3 \pm 23.7$ & Con vs. Dex & $<0.001$ \\
\hline & & & & & & Con vs. Yoh + Dex & $<0.001$ \\
\hline & & & & & & Dex vs. Yoh + Dex & 0.093 \\
\hline \multirow[t]{3}{*}{ RPP, $75 \%$ reduction } & - & - & $285.4 \pm 29.0$ & $412.6 \pm 25.1$ & $409.5 \pm 27.3$ & Con vs. Dex & $<0.001$ \\
\hline & & & & & & Con vs. Yoh + Dex & $<0.001$ \\
\hline & & & & & & Dex vs. Yoh + Dex & 0.064 \\
\hline
\end{tabular}

Tabel 1. Data were given as mean \pm SD. ( $n=8$ in each group)

${ }^{*} P<0.05$ versus Group Con. HR Heart rate, RPP Rate-pressure product, KHB Krebs-Henseleit buffer, Con Control, Dex Dexmedetomidine, Yoh Yohimbine 


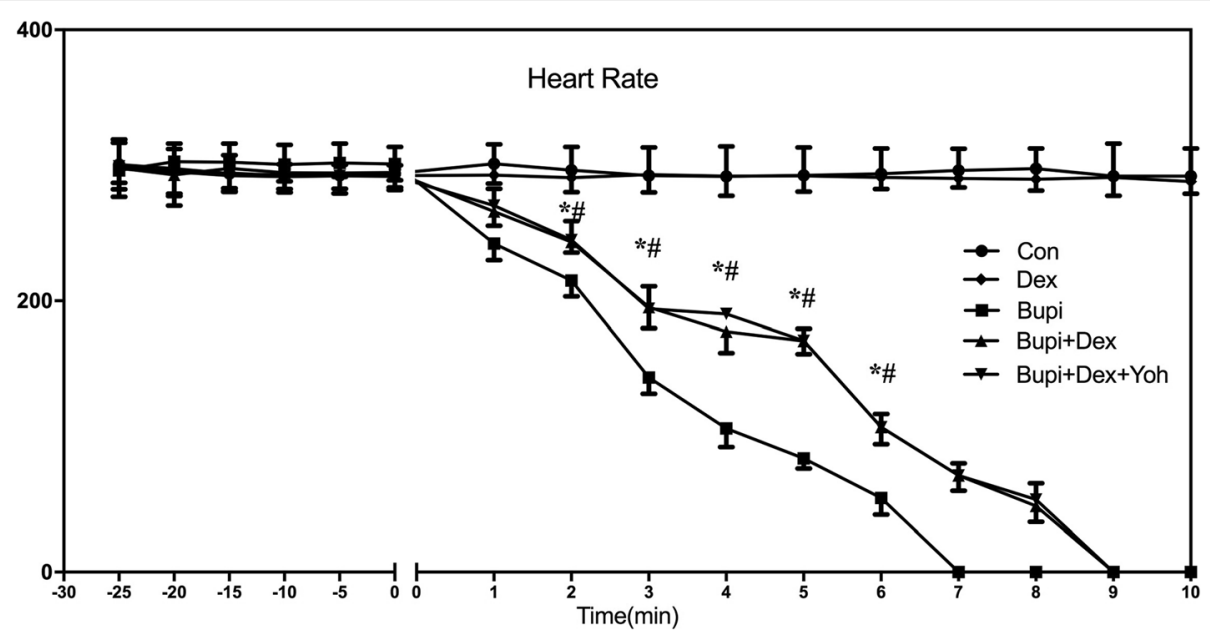

Fig. 2 Changes of HRs in five groups ( $n=8$ in each group). HR (Heart Rate) in three groups were presented graphically. Data were given as mean \pm SD. ${ }^{*} P<0.05$, between group Bupi and group Bupi + Dex, ${ }^{\sharp} P<0.05$, between group Bupi and group Bupi + Dex + Yoh. Con, control; Bupi, bupivacaine; Dex, dexmedetomidine; Yoh, yohimbine

\section{Cardiac tissue bupivacaine content}

Cardiac tissue bupivacaine content after asystole in Bupi, Bupi + Dex and Bupi + Yoh + Dex groups was $46.8 \pm 5.6 \mathrm{nmol} / \mathrm{g}, \quad 58.5 \pm 6.3 \mathrm{nmol} / \mathrm{g}$ and $57.0 \pm 6.6$ $\mathrm{nmol} / \mathrm{g}$ respectively. Cardiac tissue bupivacaine content in the Bupi + Dex and Bupi + Dex + Yoh groups was higher than that in group Bupi $(P=0.003, P=$ 0.009 , respectively, Fig. 4$)$. There was no significant difference between the Bupi + Dex and Bupi + Dex + Yoh groups $(P=0.879)$.

\section{Discussion}

Results of the present study showed as follows: (1) Dexmedetomidine had no effect on HRs and RPPs of the isolated rat hearts in group Dex. (2) Pretreatment with dexmedetomidine significantly increased the time to first

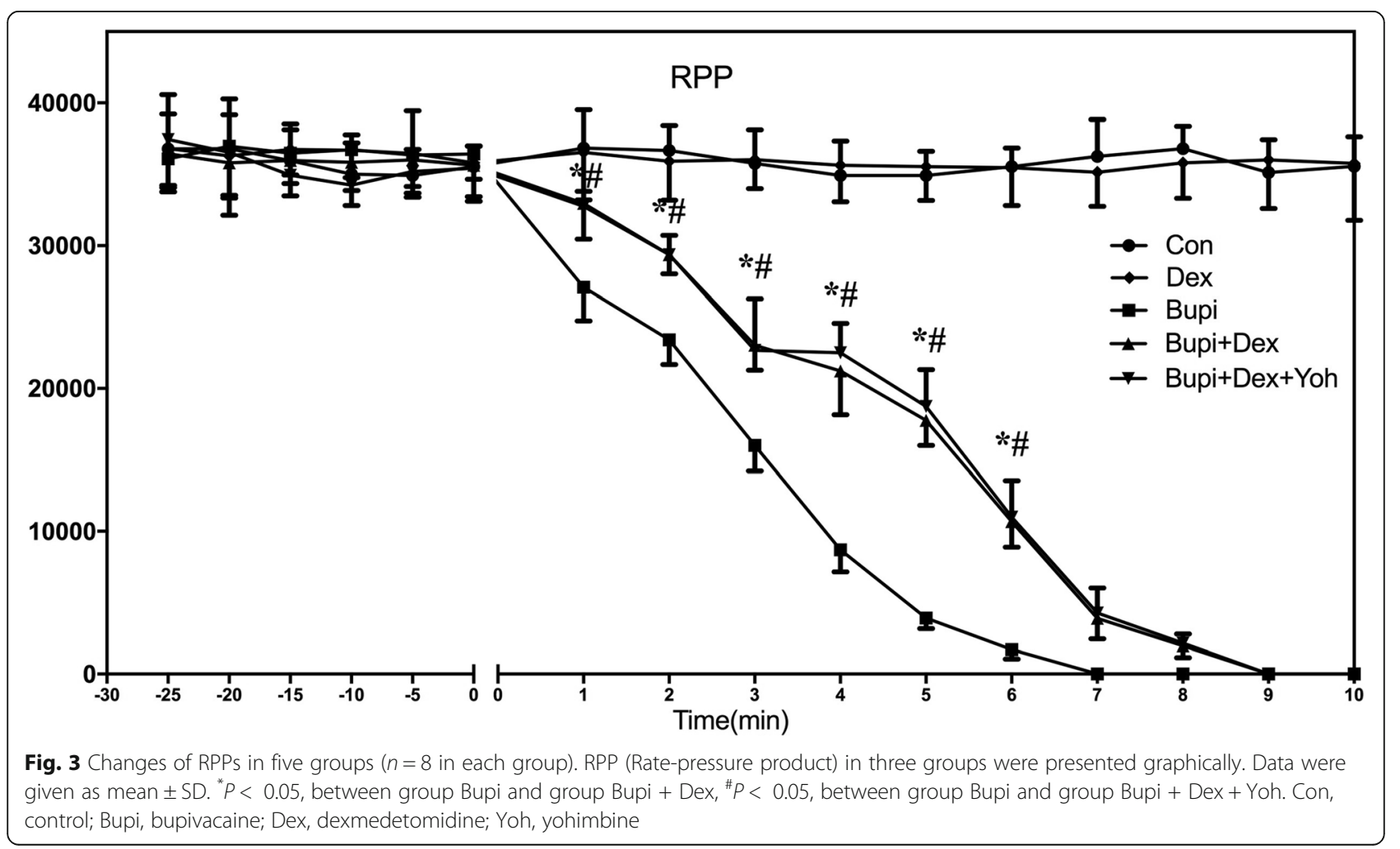




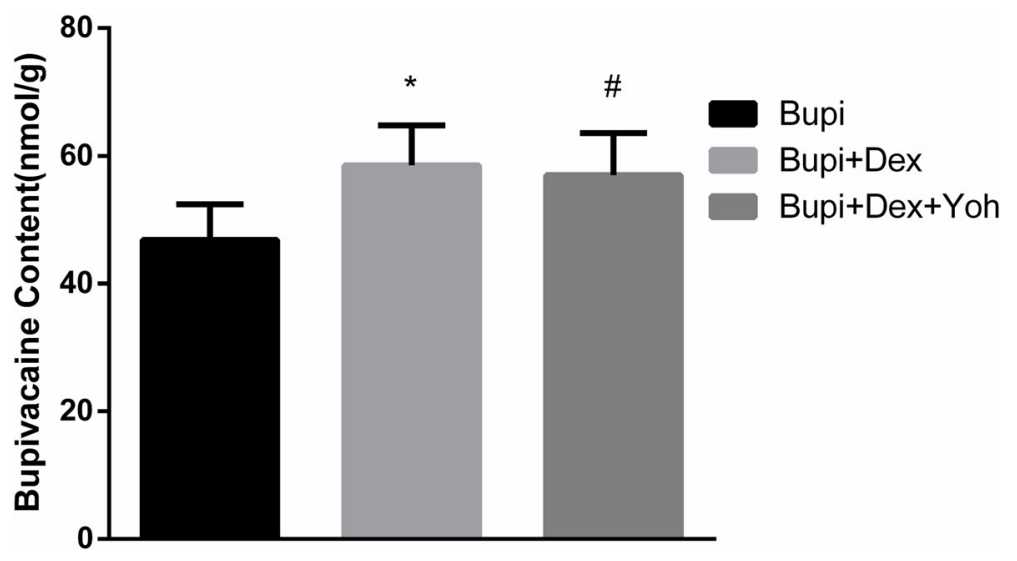

Fig. 4 Bupivacaine content in cardiac tissue among the experiment groups ( $n=8$ in each group). Data were given as mean \pm SD. ${ }^{*} P<0.05$, between group Bupi and group Bupi + Dex, ${ }^{\#} P<0.05$, between group Bupi and group Bupi + Dex + Yoh. Con, control; Bupi, bupivacaine; Dex, dexmedetomidine; Yoh, yohimbine. Bupi + Dex vs. Bupi, $P=0.003$, Bupi + Dex vs. Bupi + Dex + Yoh, $P=0.879$, Bupi + Dex + Yoh vs. Bupi, $P=0.009$

arrhythmia and time to asystole in group Bupi + Dex. (3) The times to 25,50 and $75 \%$ reductions in HR and RPP were significantly longer in the Bupi + Dex and Bupi + Dex + Yoh groups than in group Bupi. (4) Compared with group Bupi, dexmedetomidine significantly increased cardiac tissue bupivacaine content when asystole in goup Bupi + Dex and group Bupi + Dex + Yoh. (5) The benifit of dexmedetomidine in mitigating bupivacaine-induced cardiotoxicity could not be eliminated by yohimbine.

It was the first study to explore direct effects of dexmedetomidine on bupivacaine-induced cardiotoxicity in the isolated rat hearts. Compared with the model in vivo, this model could avoid the disturbance of internal environment and organ hypoxia. Based on our previous study [11, 12] and other study [13], we chose $50 \mu \mathrm{mol} / \mathrm{L}$ bupivacaine, $10 \mathrm{nmol} / \mathrm{L}$ dexmedetomidine and $1 \mu \mathrm{mol} / \mathrm{L}$ yohimbine as the optimal concentration.

Our results indicated that the cardiac function of the isolated hearts which perfused with KHB were stable throughout the experiment. Therefore, we can ignore the deterioration caused by the Langendorff heart model according the HR and RPP. Our study also showed that dexmedetomidine had no significant effect on HRs and the contractility of isolated rat hearts. In addition, Flacke et al. [14] also found in the isolated dog hearts that dexmedetomidine does not influence the heart function.

Hanci [8] proved that dexmedetomidine pretreatment delays occurrence of bupivacaine cardiotoxicity. WistarAlbino male rats were pretreated with intravenous dexmedetomidine at a dose of $10 \mu \mathrm{g} / \mathrm{kg}$ in the bupivacaine-induced cardiotoxicity. The results showed that dexmedetomidine reduced the heart rates and mean arterial pressures and increased the time of first arrhythmia and asystole in the rats. However, Hanci speculated the mechanism may be related to dexmedetomidine's sympatholytic properties and antiarrhythmogenic, without further investigation. Based on Hanci's experiment, we suspected that dexmedetomidine could alleviate bupivacaine-induced cardiotoxicity by acting directly on the isolated heart.

In this study, we found the time to arrhythmia in the Bupi + Dex group was significantly longer than that in the Bupi group, indicating that dexmedetomidine exactly has anti-arrhythmic effects. Similarly, Chrysostomou et al. [10] also found that for the patients with perioperative atrial and junctional tachyarrhythmias, dexmedetomidine may have a potential therapeutic effect on controlling the HR or conversion to normal sinus rhythm. Therefore, we suspect the anti-arrhythmic effect is also involved in dexmedetomidine's enhancing tolerance to bupivacaine cardiotoxicity in the isolated heart. However, the underlying mechanism of dexmedetomidine's anti arrhythmogenic on isolated rat heart requires further investigation.

This study also showed that the myocardial concentrations of bupivacaine when asystole was significantly higher in group Bupi + Dex than in group Bupi, indicating dexmedetomidine may enhance the tolerance to bupivacaine-induced cardiotoxicity and increase the asystole concentrations of bupivacaine in the isolated rat hearts. This result is similar to previous study [8], which proved that dexmedetomidine enhanced the tolerance to bupivacaine-induced cardiotoxicity in rats.

Alpha 2 adrenoceptor is expressed and present in cardiomyocytes [15]. It is reported that the cardioprotective effect of dexmedetomidine on the ischemia/reperfusion injury in isolated rat hearts may be mediated via alpha 2 adrenoceptor [13]. Our research showed that yohimbine had no influence on the ability of dexmedetomidine on bupivacaine-induced cardiotoxicity in the isolated hearts. In the other words, dexmedetomidine enhanced the myocardial tolerance to bupivacaine-induced cardiotoxicity 
was not associated with alpha- 2 adrenergic receptors. Accumulating evidence proves that dexmedetomidine can combine with other receptors or channels [16-18], and which suggests there may exist more mechanisms of dexmedetomidine's protective effect on bupivacaine-induced cardiotoxicity.

The mechanism underlying bupivacaine-induced arrhythmia may be the blockade of cardiac voltagegated sodium channels and L-type calcium channels, resulting in inhibition the production of the cardiac action potential [1]. The present study has demonstrated that bupivacaine causes arrhythmia or arrest. This result is similar to previous studies $[19,20]$. Besides, our previous study [21] found that bupivacaine could inhibit the opening of the mitochondrial transition pore and disturb the balance of $\mathrm{Ca}^{2+}$ in mitochondria, which ultimately caused cardiomyocyte toxicity. Therefore, we speculate the mechanism that dexmedetomidine attenuates the cardiotoxicity of bupivacaine might also involve improved function of cardiac voltage-gated sodium channels and L-type calcium channels, and mitochondrial function.

Dexmedetomidine is increasingly being used as an adjuvant to local anesthetics in regional anesthesia procedures or for intraoperative sedation in clinical. Therefore, dexmedetomidine and bupivacaine will often be used together in patients. Our results showed that dexmedetomidine can increase the heart's tolerance to bupivacaine and delay the onset of the cardiotoxicity of bupivacaine by acting directly on the heart. It suggests the use of dexmedetomidine may improve the safety use of bupivacaine in clinical, while more evidences like animal experiments and clinical studies need to be carried out.

\section{Limitations}

Compared with Hanci's study, the present study can directly reveal the effect of dexmedetomidine on the heart and the local mechanism of the ability of dexmedetomidine on bupivacaine-induced cardiotoxicity. However, it still has its limitations. First of all, we fail to explore the systemic effects of dexmedetomidine in vitro models. Besides, this study is unable to investigate the real-time concentration of myocardial bupivacaine in each group before asystole. We will design experiments to observe the impact of dexmedetomidine on the bupivacaine's distribution in the myocardium in the further study.

\section{Conclusions}

The results of the present study suggest that dexmedetomidine could enhances tolerance to bupivacaine cardiotoxicity on the isolated rat hearts, but the alpha 2 adrenoceptors were not involved in this process. Future studies should be concerned with the effects of dexmedetomidine on cardiac voltage-gated sodium channels and L-type calcium channels, and mitochondrial function.

\section{Abbreviations}

Bupi: Bupivacaine; Con: Control; Dex: Dexmedetomidine;

ECG: Electrocardiography; HR: Heart rate; KHB: Krebs-Henseleit buffer;

RPP: Rate-pressure product; $T_{\text {arrhythmia: }}$ Time to the first arrhythmia;

$T_{\text {asystole: Time to the asystole; Yoh: Yohimbine }}$

\section{Acknowledgements}

Not applicable.

\section{Author contributions}

FX, ZJ and HC designed the study, analyzed the data and wrote the manuscript. TL, XC and LP conducted the study and wrote the manuscript. SW and YC assisted with the experiments and data analysis. All authors have read and approve the final manuscript.

\section{Funding}

This work was supported by the Natural Science Foundation of Zhejiang Province, China [grant numbers LQ18H090006] and Wenzhou Municipal Scientific and Technological Program Projects [grant number Y20170042]. The Natural Science Foundation of Zhejiang Province, China [grant numbers LQ18H090006] conduct the design of the study, analyze the data and the writing the manuscript. The Wenzhou Municipal Scientific and Technological Program Projects [grant number Y20170042] conduct the collection, analysis and interpretation of data.

\section{Availability of data and materials}

The datasets used and/or analyzed during the current study are available from the corresponding author on reasonable request.

\section{Ethics approval}

All animal protocols were approved by the Animal Care and Use Committee of Wenzhou Medical University (wydw2015-0121, Zhejiang, China).

Consent for publication

Not applicable.

\section{Competing interests}

The authors declare that they have no competing interests..

Received: 13 April 2019 Accepted: 8 November 2019

Published online: 21 November 2019

\section{References}

1. Butterworth JF. Models and mechanisms of local anesthetic cardiac toxicity: a review. Reg Anesth Pain Med. 2010;35:167-76.

2. Albright GA. Cardiac arrest following regional anesthesia with etidocaine or bupivacaine. Anesthesiology. 1979;51:285-7.

3. Bhana N, Goa KL, McClellan KJ. Dexmedetomidine. Drugs. 2000;59:263-8.

4. Ebert TJ, Hall JE, Barney JA, Uhrich TD, Colinco MD. The effects of increasing plasma concentrations of dexmedetomidine in humans. Anesthesiology. 2000;93:382-94.

5. Coskuner I, Tekin M, Kati I, Yagmur C, Elcicek K. Effects of dexmedetomidine on the duration of anaesthesia and wakefulness in bupivacaine epidural block. Eur J Anaesthesiol. 2007;24:535-40.

6. Hussain N, Grzywacz VP, Ferreri CA, Atrey A, Banfield L, Shaparin N, Vydyanathan A. Investigating the efficacy of Dexmedetomidine as an adjuvant to local anesthesia in brachial plexus block: a systematic review and meta-analysis of 18 randomized controlled trials. Reg Anesth Pain Med. 2017:42:184-96.

7. Abdallah FW, Abrishami A, Brull R. The facilitatory effects of intravenous dexmedetomidine on the duration of spinal anesthesia: a systematic review and meta-analysis. Anesth Analg. 2013;117:271-8.

8. Hanci V, Karakaya K, Yurtlu S, Hakimoglu S, Can M, Ayoglu H, Erdogan G, Okyay RD, Turan 1O. Effects of dexmedetomidine pretreatment on bupivacaine cardiotoxicity in rats. Reg Anesth Pain Med. 2009;34:565-8.

9. Willigers HM, Prinzen FW, Roekaerts PM, de Lange S, Durieux ME. Dexmedetomidine decreases perioperative myocardial lactate release in dogs. Anesth Analg. 2003;96:657-64.

10. Chrysostomou C, Beerman L, Shiderly D, Berry D, Morell VO, Munoz R. Dexmedetomidine: a novel drug for the treatment of atrial and junctional 
tachyarrhythmias during the perioperative period for congenital cardiac surgery: a preliminary study. Anesth Analg. 2008;107:1514-22.

11. Liu L, Xia Y, Chen Y, Wang Q, Shi T, Wang F, Small RH, Xu X. The comparative effects of lipid, epinephrine, and their combination in the reversal of bupivacaine-induced asystole in the isolated rat heart. Anesth Analg. 2012;114:886-93.

12. Chen Y, Xia Y, Liu L, Shi T, Shi K, Wang Q, Chen L, Papadimos TJ, Xu X. Lipid emulsion reverses bupivacaine-induced asystole in isolated rat hearts: concentration-response and time-response relationships. Anesthesiology 2010;113:1320-5.

13. Okada H, Kurita T, Mochizuki T, Morita K, Sato S. The cardioprotective effect of dexmedetomidine on global ischaemia in isolated rat hearts. Resuscitation. 2007;74:538-45.

14. Flacke WE, Flacke JW, Blow KD, McIntee DF, Bloor BC. Effect of dexmedetomidine, an alpha 2-adrenergic agonist, in the isolated heart. J Cardiothorac Vasc Anesth. 1992;6:418-23.

15. Dukes ID, Vaughan Williams EM. Effects of selective alpha 1-, alpha 2-, beta 1-and beta 2-adrenoceptor stimulation on potentials and contractions in the rabbit heart. J Physiol. 1984;355:523-46.

16. Behmenburg F, Pickert E, Mathes A, Heinen A, Hollmann MW, Huhn R, Berger MM. The Cardioprotective effect of Dexmedetomidine in rats is dose-dependent and mediated by BKCa channels. J Cardiovasc Pharmacol. 2017;69:228-35.

17. Stoetzer C, Reuter S, Doll T, Foadi N, Wegner F, Leffler A. Inhibition of the cardiac $\mathrm{Na}(+)$ channel alpha-subunit Nav1.5 by propofol and dexmedetomidine. Naunyn Schmiedeberg's Arch Pharmacol. 2016;389:315-25.

18. Sun Y, Jiang C, Jiang J, Qiu L. Dexmedetomidine protects mice against myocardium ischaemic/reperfusion injury by activating an AMPK/PI3K/Akt/ eNOS pathway. Clin Exp Pharmacol Physiol. 2017;44:946-53.

19. Simon L, Kariya N, Pelle-Lancien E, Mazoit JX. Bupivacaine-induced QRS prolongation is enhanced by lidocaine and by phenytoin in rabbit hearts. Anesth Analg. 2002;94:203-7.

20. Simon L, Kariya N, Edouard A, Benhamou D, Mazoit JX. Effect of bupivacaine on the isolated rabbit heart: developmental aspect on ventricular conduction and contractility. Anesthesiology. 2004;101:937-44.

21. Chen Z, Jin Z, Xia Y, Zhao S, Xu X, Papadimos TJ, Wang Q. The protective effect of lipid emulsion in preventing bupivacaine-induced mitochondrial injury and apoptosis of H9C2 cardiomyocytes. Drug Deliv. 2017;24:430-6.

\section{Publisher's Note}

Springer Nature remains neutral with regard to jurisdictional claims in published maps and institutional affiliations.

Ready to submit your research? Choose BMC and benefit from:

- fast, convenient online submission

- thorough peer review by experienced researchers in your field

- rapid publication on acceptance

- support for research data, including large and complex data types

- gold Open Access which fosters wider collaboration and increased citations

- maximum visibility for your research: over $100 \mathrm{M}$ website views per year

At $\mathrm{BMC}$, research is always in progress.

Learn more biomedcentral.com/submissions 